\title{
ON SOLUTIONS OF SEMILINEAR EVOLUTION INTEGRODIFFERENTIAL EQUATIONS WITH NONLOCAL CONDITIONS
}

\author{
ZUOMAO YAN
}

\begin{abstract}
In this paper, by using the theory of evolution families, Banach's contraction principle and Schauder's fixed point theorem, we prove the existence of mild solutions of a class of semilinear evolution integrodifferential equations with nonlocal conditions in Banach space. An example is provided to illustrate the obtained results.
\end{abstract}

\section{Introduction}

In this paper, we study the existence of solutions for semilinear evolution integrodifferential equations with nonlocal conditions of the following form:

$$
\begin{aligned}
& x^{\prime}(t)=A(t) x(t)+F\left(t, x(t), \int_{0}^{t} h(t, s, x(s)) d s\right), \quad t \in J=[0, b], \\
& x(0)+g(x)=x_{0},
\end{aligned}
$$

where the family $\{A(t): 0 \leq t \leq b\}$ of linear operator generates a linear evolution systems. The nonlinear operators $F: J \times X \times X \rightarrow X, h: J \times J \times X \rightarrow X$ and $g: C(J, X) \rightarrow \overline{D(A)}$ are given funnctions.

The abstract nonlocal problem was first studied by Byszewski [1], and subsequently, as it can be applied in physics with better than the classical initial condition, it has been studied extensively. Byszewski and Akca [2] established the existence of mild and classical solutions of nonlocal Cauchy problem for semilinear functional differential evolution equation with the help of Schauder's fixed point theorem. Ntouyas and Tsamatos [3] studied the global existence of solution for semilinear evolution equations with nonlocal conditions by using the Leray-Schauder Alternative. In [4], Lin and Liu discussed the existence of mild solution for autonomous semilinear integrodifferential equations under Lipschitz-type conditions. Ezzinbi and Fu [5] studied the existence and regularity of solutions for some neutral partial differential equations with nonlocal conditions. Recently,

Received January 22, 2008.

2000 Mathematics Subject Classification. 34K30, 34G20, 47B38.

Key words and phrases. Semilinear evolution integrodifferential equations, linear evolution system, fixed point theorem, nonlocal conditions. 
Liang et al. [6] proved the existence of mild solutions for the nonlinear nonlocal Cauchy problem, and give a related condition for a general mapping $g$. Aizicovici and Lee [7] investigated the existence of integral solutions to the associated nonlocal problem, where $A(t)=A$ is an $m$-accretive operators in $X$ generating a compact nonlinear contraction semigroup. In paper [8] the authors also obtained the existence of integral solutions to the associated nonlocal problem, where $\{A(t)\}_{0 \leq t \leq b}$ is a family of $m$-accretive operators in $X$ generating a compact evolution family. Sakthivel et al. [9] have shown that the existence of mild solutions for a class of nonautonomous semilinear evolution integrodifferential systems in Banach spaces, where $A(t)$ is a closed linear operator on $X$ with dense domain $D(A)$ which is independent of $t$. The main tools and techniques in [9] are the properties of resolvent operators and Banach's contraction principle. In this paper, our main approach has two wedges, on one hand, the $g$ is continuous but without impose severe compactness condition and the convexity. Further, $g$ is not assumed to be Lipschitz. On the other hand, we shall investigate the existence of mild solutions of nonlocal Cauchy problem (1.1) in Banach spaces, by means of a different method, that is, by using the theory of evolution families, Banach's contraction principle and Schauder's fixed point theorem.

This paper will be organized as follows. In Section 2 give some preliminaries about the theory of evolution families which will be used in paper. Section 3 is devoted to the existence of mild solutions of equations (1.1). Further, we present existence results for the nonlocal problem (1.1) for a spacial case. Finally, a concrete example is presented in Section 4 to show the applications of our main results.

\section{Preliminaries}

Let $X$ be a Banach space endowed with the norm $\|\cdot\|$ and the family $\{A(t): 0 \leq t \leq b\}$ of linear operators, we need the following assumptions (see [10]).

(I) the domain $D(A)$ of $\{A(t): 0 \leq t \leq b\}$ is dense in the Banach space $X$ and independent of $t, A(t)$ is a closed linear operator.

(II) For each $t \in J$, the resolvent $R(\lambda, A(t))$ exists for all $\lambda$ with $\operatorname{Re} \lambda \geq 0$ and there exists $K>0$ such that

$$
\|R(\lambda, A(t))\| \leq \frac{K}{(|\lambda|+1)}
$$

(III) For any $t, s, \tau \in J$, there exists a $0<\beta<1$ and $K>0$ such that

$$
\left\|(A(t)-A(\tau)) A^{-1}(s)\right\| \leq K|t-\tau|^{\beta} .
$$

And for each $t \in J$ and some $\lambda \in \rho(A(t))$, the resolvent $R(\lambda, A(t))$ set of $A(t)$ is a compact operator.

If conditions (I)-(III) are satisfied, then the family $\{A(t): 0 \leq t \leq b \mid\}$ generates a unique linear evolution system $\{U(t, s): 0 \leq s \leq t \leq b\}$ satisfying the following properties: (see [11]) 
(a) $U(t, s) \in L(X)$ the space of bounded linear transformations of $X$ whenever $0 \leq s \leq$ $t \leq b$ and for each $x \in X$ the mapping $(t, s) \rightarrow U(t, s) x$ is continuous;

(b) $U(t, s) U(s, \tau)=U(t, \tau)$ whenever $0 \leq \tau \leq s \leq t \leq b$;

(c) $U(t, t)=I$, the identity operator on $X$, for each $t \in J$;

(d) $U(t, s)$ is a compact linear operator on $X$ wherever $t-s>0(0 \leq s<t \leq b)$.

For the evolution system $\{U(t, s): 0 \leq s \leq t \leq b\}$, the following properties and proposition are well known and we will need them in paper.

(e) There exist constant $M>0$, such that $\|U(t, s)\| \leq M, 0 \leq s<t \leq b$.

Proposition 2.1. The family operators $\{U(t, s), t>s\}$ is continuous in $t$ in the uniform operator topology uniformly for $s$.

Definition 2.1. A continuous function $x(\cdot): J \rightarrow X$ is a said to be a mild solution to problem (1.1) if for all $x_{0} \in X$, it satisfies the following integral equation:

$$
x(t)=U(t, 0)\left[x_{0}-g(x)\right]+\int_{0}^{t} U(t, s) F\left(s, x(s), \int_{0}^{s} h(s, \tau, x(\tau)) d \tau\right) d s .
$$

Further we assume the following hypotheses:

(H1) The function $F: J \times X \times X \rightarrow X$ is continuous and there exist constants $L>0$, $L_{1}>0$, such that for all $x_{i}, y_{i} \in X, i=1,2$, we have

$$
\left\|F\left(t, x_{1}, y_{1}\right)-F\left(t, x_{2}, y_{2}\right)\right\| \leq L\left[\left\|x_{1}-x_{2}\right\|+\left\|y_{1}-y_{2}\right\|\right],
$$

and

$$
L_{1}=\max _{t \in J}\|F(t, 0,0)\| .
$$

(H2) The function $h: J \times J \times X \rightarrow X$ is continuous and there exist constants $N>0$, $N_{1}>0$, such that for all $x_{i} \in X, i=1,2$,

$$
\left\|h\left(t, s, x_{1}\right)-h\left(t, s, x_{2}\right)\right\| \leq N\left\|x_{1}-x_{2}\right\|,
$$

and

$$
N_{1}=\max _{0 \leq s \leq t \leq b}\|h(t, s, 0)\| .
$$

(H3) The function $g(\cdot): C(J, X) \rightarrow \overline{D(A)}$ is continuous and there exists a $\delta \in(0, b)$ such that $g(\phi)=g(\psi)$ for any $\phi, \psi \in C:=C(J, X)$ with $\phi=\psi$ on $[\delta, b]$; In addition, there is a continuous nondecreasing function $\Lambda:[0, \infty) \rightarrow(0, \infty)$ such that

$$
\|g(\phi)\| \leq \Lambda(\|\phi\|), \quad \phi \in C,
$$

and for $\rho>0$

$$
\lim _{\rho \rightarrow \infty} \sup \frac{\Lambda(\rho)}{\rho}=\gamma<\infty
$$




\section{Main Result}

Theorem 3.1. Let $x_{0} \in \overline{D(A)}$. Assume that assumptions (H1)-(H3) hold. Also assume that

$$
M e^{b M L(1+N b)} \gamma<1 \text {. }
$$

Then the nonlocal Cauchy problem (1.1) has at least one mild solution on J.

Proof. Consider the space $C:=C(J, X)$ the Banach space of all continuous functions from $J$ to $X$ endowed with sup norm.

Let $L_{0}:=2 M L(1+N b)$ and we introduce in the space $C$ the equivalent norm defined as

$$
\|\phi\|_{V}:=\sup _{t \in J} e^{-L_{0} t}\|\phi(t)\| .
$$

Then, it is easy to see that $V:=\left(C(J, X),\|\cdot\|_{V}\right)$ is a Banach space. Fix $v \in C$ and for $t \in J, \phi \in V$, we now defined an operator

$$
\left(Q_{v} \phi\right)(t)=U(t, 0)\left[x_{0}-g(v)\right]+\int_{0}^{t} U(t, s) F\left(s, \phi(s), \int_{0}^{s} h(s, \tau, \phi(\tau)) d \tau\right) d s .
$$

Since $U(\cdot, 0)\left(x_{0}-g(v)\right) \in C(J, X)$, so, it follows from (a), (e) that $\left(Q_{v} \phi\right)(t) \in V$ for all $\phi \in V$. Let $\phi, \psi \in V$, we have

$$
\begin{aligned}
e^{-L_{0} t} & \left\|\left(Q_{v} \phi\right)(t)-\left(Q_{v} \psi\right)(t)\right\| \\
\leq & e^{-L_{0} t} \int_{0}^{t}\|U(t, s)\| \| F\left(s, \phi(s), \int_{0}^{s} h(s, \tau, \phi(\tau)) d \tau\right) \\
& -F\left(s, \psi(s), \int_{0}^{s} h(s, \tau, \psi(\tau)) d \tau\right) \| d s \\
\leq & M L \int_{0}^{t} e^{-L_{0} t}\left[\|\phi(s)-\psi(s)\|+\left\|\int_{0}^{s} h(s, \tau, \phi(\tau)) d \tau-\int_{0}^{s} h(s, \tau, \psi(\tau)) d \tau\right\|\right] d s \\
\leq & M L \int_{0}^{t} e^{L_{0}(s-t)}\left[e^{-L_{0} s}\|\phi(s)-\psi(s)\|+N b \sup _{s \in J} e^{-L_{0} s}\|\phi(s)-\psi(s)\|\right] d s \\
\leq & M L(1+N b) \int_{0}^{t} e^{L_{0}(s-t)} d s\|\phi-\psi\|_{V} \\
\leq & \frac{M L(1+N b)}{L_{0}}\|\phi-\psi\|_{V}, \quad t \in J
\end{aligned}
$$

which implies that

$$
e^{-L_{0} t}\left\|\left(Q_{v} \phi\right)(t)-\left(Q_{v} \psi\right)(t)\right\| \leq \frac{1}{2}\|\phi-\psi\|_{V}, \quad t \in J .
$$

Thus

$$
\left\|Q_{v} \phi-Q_{v} \psi\right\|_{V} \leq \frac{1}{2}\|\phi-\psi\|_{V}, \quad \phi, \psi \in V
$$


Therefore, $Q_{v}$ is a strict contraction. By Banach's contraction principle we conclude that $Q_{v}$ has a unique fixed point $\phi_{v} \in V$ and Eq. (3.2) has a unique mild solution on $[0, b]$. Now let $r>0$ and

$$
v \in C_{r}(\delta):=\left\{\phi \in C([\delta, b], X) ; \quad \sup _{\delta \leq t \leq b}\|\phi(t)\| \leq r\right\} .
$$

Set

$$
\tilde{v}(t):= \begin{cases}v(t) & \text { if } t \in(\delta, b] \\ v(\delta) & \text { if } t \in[0, \delta]\end{cases}
$$

From (3.2), we have

$$
\phi_{\tilde{v}}(t)=U(t, 0)\left[x_{0}-g(\tilde{v})\right]+\int_{0}^{t} U(t, s) F\left(s, \phi_{\tilde{v}}(s), \int_{0}^{s} h\left(s, \tau, \phi_{\tilde{v}}(\tau)\right) d \tau\right) d s .
$$

Then, for $t \in(0, b]$,

$$
\begin{aligned}
\left\|\phi_{\tilde{v}}(t)\right\| \leq & \left\|U(t, 0)\left[x_{0}-g(\tilde{v})\right]\right\|+\int_{0}^{t}\left\|U(t, s) F\left(s, \phi_{\tilde{v}}(s), \int_{0}^{s} h\left(s, \tau, \phi_{\tilde{v}}(\tau)\right) d \tau\right)\right\| d s \\
\leq & \|U(t, 0)\|\left\|x_{0}-g(\tilde{v})\right\|+\int_{0}^{t}\|U(t, s)\|\left[\| F\left(s, \phi_{\tilde{v}}(s), \int_{0}^{s} h\left(s, \tau, \phi_{\tilde{v}}(\tau)\right) d \tau\right)\right. \\
& -F(s, 0,0)\|+\| F(s, 0,0) \|] d s \\
\leq & M\left[\left\|x_{0}\right\|+\|g(\tilde{v})\|\right]+M \int_{0}^{t}\left\{L\left[\left\|\phi_{\tilde{v}}(s)\right\|+\left\|\int_{0}^{s} h(s, \tau, \phi(\tau)) d \tau\right\|\right]+L_{1}\right\} d s \\
\leq & M\left[\left\|x_{0}\right\|+\Lambda(r)\right]+M \int_{0}^{t}\left\{L \left[\left\|\phi_{\tilde{v}}(s)\right\|+\int_{0}^{s}\left[\left\|h\left(s, \tau, \phi_{\tilde{v}}(\tau)\right)-h(s, \tau, 0)\right\|\right.\right.\right. \\
& \left.+\|h(s, \tau, 0)\|] d \tau]+L_{1}\right\} d s \\
\leq & M\left[\left\|x_{0}\right\|+\Lambda(r)\right]+M \int_{0}^{t}\left\{L\left[\left\|\phi_{\tilde{v}}(s)\right\|+b\left[N \sup _{s \in(0, b]}^{t}\left\|\phi_{\tilde{v}}(s)\right\|+N_{1}\right]\right]+L_{1}\right\} d s \\
\leq & M\left[\left\|x_{0}\right\|+\Lambda(r)+L b N_{1}+b L_{1}\right]+M L(1+N b) \int_{0}^{t} \sup _{s \in(0, b]}\left\|\phi_{\tilde{v}}(s)\right\| d s .
\end{aligned}
$$

Making use of the Gronwall's inequality, such that

$$
\sup _{t \in(0, b]}\left\|\phi_{\tilde{v}}(t)\right\| \leq M\left[\left\|x_{0}\right\|+\Lambda(r)+L b N_{1}+b L_{1}\right] e^{b M L(1+N b)} .
$$

Next we claim that $\phi_{\tilde{v}}(t) \in C_{r}(\delta)$ for $v \in C_{r}(\delta)$. If it is not true, then for some positive number $r$, there is a function $v \in C_{r}(\delta)$, but $\phi_{\tilde{v}}(t) \notin C_{r}(\delta)$, that is, $\sup _{t \in[\delta, b]}\left\|\phi_{\tilde{v}}(t)\right\|>r$ for 
some $t \in(0, b]$. However, on the other hand, we have

$$
\begin{aligned}
r & <\sup _{t \in[\delta, b]}\left\|\phi_{\tilde{v}}(t)\right\| \\
& \leq M\left[\left\|x_{0}\right\|+\Lambda(r)+L b N_{1}+b L_{1}\right] e^{b M L(1+N b)} .
\end{aligned}
$$

Thus

$$
1<\frac{1}{r} M\left[\left\|x_{0}\right\|+L b N_{1}+b L_{1}\right] e^{b M L(1+N b)}+M e^{b M L(1+N b)} \frac{\Lambda(r)}{r} .
$$

Using (H3) and $r$ enough large we conclude that

$$
M e^{b M L(1+N b)} \gamma>1
$$

which is contradicts (3.1). Hence there exists a positive noumber $r, \phi_{\tilde{v}}(t) \in C_{r}(\delta)$ for $v \in C_{r}(\delta)$.

Based on this fact, we will show a mapping $P: C_{r}(\delta) \rightarrow C_{r}(\delta)$ definde by

$$
(P v)(t)=\phi_{\tilde{v}}(t), \quad t \in[\delta, b] .
$$

From $(3.3),(\mathrm{H} 1)$ and (H2), we deduce that for $v_{1}, v_{2} \in C_{r}(\delta), t \in[0, b]$,

$$
\begin{aligned}
\left\|\phi_{\tilde{v}_{1}}(t)-\phi_{\tilde{v}_{2}}(t)\right\| & \\
\leq & \|U(t, 0)\|\left\|g\left(\tilde{v}_{1}\right)-g\left(\tilde{v}_{2}\right)\right\|+\int_{0}^{t}\|U(t, s)\| \| F\left(s, \phi_{\tilde{v}_{1}}(s), \int_{0}^{s} h\left(s, \tau, \phi_{\tilde{v}_{1}}(\tau)\right) d \tau\right) \\
& \quad-F\left(s, \phi_{\tilde{v}_{2}}(s), \int_{0}^{s} h\left(s, \tau, \phi_{\tilde{v}_{2}}(\tau)\right) d \tau\right) \| d s \\
\leq & M\left\|g\left(\tilde{v}_{1}\right)-g\left(\tilde{v}_{2}\right)\right\|+M L \int_{0}^{t}\left[\left\|\phi_{\tilde{v}_{1}}(s)-\phi_{\tilde{v}_{2}}(s)\right\|+\| \int_{0}^{s} h\left(s, \tau, \phi_{\tilde{v}_{1}}(\tau)\right) d \tau\right. \\
& \left.\quad-\int_{0}^{s} h\left(s, \tau, \phi_{\tilde{v}_{2}}(\tau)\right) d \tau \|\right] d s \\
\leq & M\left\|g\left(\tilde{v}_{1}\right)-g\left(\tilde{v}_{2}\right)\right\|+M L \int_{0}^{t}\left[\left\|\phi_{\tilde{v}_{1}}(s)-\phi_{\tilde{v}_{2}}(s)\right\|+N b \sup _{s \in J}\left\|\phi_{\tilde{v}_{1}}(s)-\phi_{\tilde{v}_{2}}(s)\right\|\right] d s \\
\leq & M\left\|g\left(\tilde{v}_{1}\right)-g\left(\tilde{v}_{2}\right)\right\|+M L(1+N b) \int_{0}^{t} \sup _{s \in J}\left\|\phi_{\tilde{v}_{1}}(s)-\phi_{\tilde{v}_{2}}(s)\right\| d s .
\end{aligned}
$$

Using again the Gronwall's inequality, that for $t, v_{1}, v_{2}$ as above

$$
\sup _{t \in J}\left\|\phi_{\tilde{v}_{1}}(t)-\phi_{\tilde{v}_{2}}(t)\right\| \leq M e^{M L b(1+N b)}\left\|g\left(\tilde{v}_{1}\right)-g\left(\tilde{v}_{2}\right)\right\|,
$$

for all $t \in[\delta, b], v_{1}, v_{2} \in C_{r}(\delta)$, which implies that

$$
\left\|P v_{1}-P v_{2}\right\| \leq M e^{M L b(1+N b)}\left\|g\left(\tilde{v}_{1}\right)-g\left(\tilde{v}_{2}\right)\right\| .
$$


Therefore, $P$ is continuous.

Next we will prove that the $P$ has a fixed point on $C_{r}(\delta)$, which implies equation (2.1) has a mild solution. To this end, we will show that the family $\left\{P v: v \in C_{r}(\delta)\right\}$ is family of equicontinuous functions. Let $\delta \leq t_{1} \leq t_{2} \leq b$, we have

$$
\begin{aligned}
& \left\|P v\left(t_{2}\right)-P v\left(t_{1}\right)\right\| \leq\left\|\left[U\left(t_{2}, 0\right)-U\left(t_{1}, 0\right)\right]\left[x_{0}-g(\tilde{v})\right]\right\| \\
& +\int_{0}^{t_{1}}\left\|\left[U\left(t_{2}, s\right)-U\left(t_{1}, s\right)\right] F\left(s, \phi_{\tilde{v}}(s), \int_{0}^{s} h\left(s, \tau, \phi_{\tilde{v}}(\tau)\right) d \tau\right)\right\| d s \\
& +\int_{t_{1}}^{t_{2}}\left\|U\left(t_{2}, s\right)\right\|\left\|F\left(s, \phi_{\tilde{v}}(s), \int_{0}^{s} h\left(s, \tau, \phi_{\tilde{v}}(\tau)\right) d \tau\right)\right\| d s \\
& \leq\left\|U\left(t_{2}, 0\right)-U\left(t_{1}, 0\right)\right\|\left[\left\|x_{0}\right\|+\Lambda(r)\right] \\
& +\int_{0}^{t_{1}}\left\|U\left(t_{2}, s\right)-U\left(t_{1}, s\right)\right\|\left\|F\left(s, \phi_{\tilde{v}}(s), \int_{0}^{s} h\left(s, \tau, \phi_{\tilde{v}}(\tau)\right) d \tau\right)\right\| d s \\
& +M \int_{t_{1}}^{t_{2}}\left\|F\left(s, \phi_{\tilde{v}}(s), \int_{0}^{s} h\left(s, \tau, \phi_{\tilde{v}}(\tau)\right) d \tau\right)\right\| d s .
\end{aligned}
$$

Noting that

$$
\begin{aligned}
\| F & \left(s, \phi_{\tilde{v}}(s), \int_{0}^{s} h\left(s, \tau, \phi_{\tilde{v}}(\tau)\right) d \tau\right) \| \\
& \leq\left\|F\left(s, \phi_{\tilde{v}}(s), \int_{0}^{s} h\left(s, \tau, \phi_{\tilde{v}}(\tau)\right) d \tau\right)-F(s, 0,0)\right\|+\|F(s, 0,0)\| \\
& \leq L\left[\left\|\phi_{\tilde{v}}(s)\right\|+\left\|\int_{0}^{s} h\left(s, \tau, \phi_{\tilde{v}}(\tau)\right) d \tau\right\|\right]+L_{1} \\
& \leq L\left[\left\|\phi_{\tilde{v}}(s)\right\|+\int_{0}^{s}\left[\left\|h\left(s, \tau, \phi_{\tilde{v}}\right)-h(s, \tau, 0)\right\|+\|h(s, \tau, 0)\|\right] d \tau\right]+L_{1} \\
& \leq L\left[\left\|\phi_{\tilde{v}}(s)\right\|+b\left[N \sup _{s \in[\delta, b]}\left\|\phi_{\tilde{v}}(s)\right\|+N_{1}\right]\right]+L_{1} \\
& \leq L\left[(1+N b) \sup _{s \in[\delta, b]}\left\|\phi_{\tilde{v}}(s)\right\|+N_{1}\right]+L_{1} \\
& \leq L\left[(1+N b) r+b N_{1}\right]+L_{1} .
\end{aligned}
$$

We see that $\left\|P v\left(t_{2}\right)-P v\left(t_{1}\right)\right\|$ tend to zero independently of $v \in C_{r}(\delta)$ tend to zero as $t_{2}-t_{1} \rightarrow 0$, since Proposition 2.1 which implies the family operators $\{U(t, s), t>s\}$ is continuous in $t$ in the uniform operator topology uniformly for $s$. Hence, the family of functions $\left\{\left(P_{v}\right): v \in C_{r}(\delta)\right\}$ is equicontinuous on $[\delta, b]$. 
It remains to show that $P\left(C_{r}(\delta)\right)$ is a precompact subset of $C_{r}(\delta)$.

Let $\delta<t \leq s \leq b$ be fixed and $\varepsilon$ a real number satisfying $0<\varepsilon<t$, for $v \in C_{r}(\delta)$, we define

$$
\left(P_{\varepsilon} v\right)(t)=U(t, 0)\left[x_{0}-g(v)\right]+\int_{0}^{t-\varepsilon} U(t, s) F\left(s, \phi_{\tilde{v}}(s), \int_{0}^{s} h\left(s, \tau, \phi_{\tilde{v}}(\tau)\right) d \tau\right) d s .
$$

Using the compactness of $U(t, s)(t-s>0)$, we obtain the set $\left\{P_{\varepsilon} v(t): v \in Y_{r}(\delta)\right\}$ is precompact $v \in C_{r}(\delta)$ for every $\varepsilon, 0<\varepsilon<t$. Moreover for every $v \in C_{r}(\delta)$ we have

$$
\begin{aligned}
\left\|(P v)(t)-P_{\varepsilon} v(t)\right\| \\
\leq \int_{t-\varepsilon}^{t}\|U(t, s)\|\left\|F\left(s, \phi_{\tilde{v}}(s), \int_{0}^{s} h\left(s, \tau, \phi_{\tilde{v}}(\tau)\right) d \tau\right)\right\| d s \\
\leq M \int_{t-\varepsilon}^{t}\left[\left\|F\left(s, \phi_{\tilde{v}}(s), \int_{0}^{s} h\left(s, \tau, \phi_{\tilde{v}}(\tau)\right) d \tau\right)-F(s, 0,0)\right\|+\|F(s, 0,0)\|\right] d s \\
\leq M \int_{t-\varepsilon}^{t}\left\{L\left[\left\|\phi_{\tilde{v}}(s)\right\|+\left\|\int_{0}^{s} h\left(s,, \tau, \phi_{\tilde{v}}(\tau)\right) d \tau\right\|\right]+L_{1}\right\} d s \\
\leq M \int_{t-\varepsilon}^{t}\left\{L \left[\left\|\phi_{\tilde{v}}(s)\right\|+\int_{0}^{s}\left[\left\|h\left(s, \tau, \phi_{\tilde{v}}(\tau)\right)-h(s, \tau, 0)\right\|\right.\right.\right. \\
\left.\quad+\|h(s, \tau, 0)\|] d \tau]+L_{1}\right\} d s \\
\leq M \int_{t-\varepsilon}^{t}\left\{L\left[\left\|\phi_{\tilde{v}}(s)\right\|+b\left[N \sup _{s \in[\delta, b]}\left\|\phi_{\tilde{v}}(s)\right\|+N_{1}\right]\right]+L_{1}\right\} d s \\
\leq M \int_{t-\varepsilon}^{t}\left[L\left(1+N b \sup _{s \in[\delta, b]}\left\|\phi_{\tilde{v}}(s)\right\|+L N_{1}+L_{1}\right] d s\right. \\
\leq M\left[L(1+N b) r+L N_{1}+L_{1}\right] \varepsilon .
\end{aligned}
$$

Therefore there are precompact sets arbitrarily close to the set $\left\{(P v): v \in C_{r}(\delta)\right\}$. Hence the set $\left\{(P v): v \in C_{r}(\delta)\right\}$ is a precompact in $X$. These arguments enable us to conclude that $P$ is completely continuous. We can now apply Schauder's fixed point theorem to conclude that $P$ has at least fixed point $\tilde{v}_{*} \in C_{r}(\delta)$. Let $x=\phi_{\tilde{v}_{*}}$. Then, we have

$$
x(t)=U(t, 0)\left[x_{0}-g\left(\tilde{v}_{*}\right)\right]+\int_{0}^{t} U(t, s) F\left(s, x(s), \int_{0}^{s} h(s, \tau, x(\tau)) d \tau\right) d s .
$$

Noting that $x=\phi_{\tilde{v}}-\left(P_{\tilde{v}_{*}}\right)(t)=\tilde{v}_{*}, t \in[\delta, b]$. By (H3), we obtain

$$
g(x)=g\left(\tilde{v}_{*}\right) .
$$

This implies that $x$ is an mild solution of the nonlocal Cauchy problem (1.1), and completes the proof of Theorem 3.1 . 
At last, we discuss a special case of the nonlocal condition, we propose the following equation:

$$
\begin{gathered}
x^{\prime}(t)=A(t) x(t)+F\left(t, x(t), \int_{0}^{t} h(t, s, x(s)) d s\right), \quad t \in J=[0, b], \\
x(0)+\sum_{i=0}^{p} g_{i}\left(x\left(t_{i}\right)\right)=x_{0},
\end{gathered}
$$

where $p \in \mathrm{N}^{+}, 0<t_{1}<\cdots<t_{p} \leq b$.

We suppose the frllowing conditions:

(H4) (i) The function $g_{i}(\cdot): X \rightarrow \overline{D(A)}$ is continuous and there exists a $\delta \in(0, b)$ such that $g_{i}(\phi)=g_{i}(\psi)$ for any $\phi, \psi \in C:=C(J, X)$ with $\phi=\psi$ on $[\delta, b]$.

(ii) There is a continuous nondecreasing function $\Lambda_{i}:[0, \infty) \rightarrow(0, \infty)$ such that

$$
\left\|g_{i}(\phi)\right\| \leq \Lambda_{i}(\|\phi\|), \quad \phi \in C
$$

and for $\rho>0$

$$
\lim _{\rho \rightarrow \infty} \sup \frac{\Lambda_{i}(\rho)}{\rho}=0
$$

Theorem 3.2. Let $x_{0} \in \overline{D(A)}$. Assume that assumptions $(\mathrm{H} 1),(\mathrm{H} 2)$ and $(\mathrm{H} 4)$ hold. Then the nonlocal Cauchy problem (3.9) has at least one mild solution.

Proof. Let $L_{0}, C, V, Q_{v}, \tilde{v}$ as in Theorem 3.1. Therefore, $Q_{v}$ has a unique fixed point $\phi(t, v) \in V$ and Eq. (3.2) has a unique solution $\phi(t, v)$ such that for $0 \leq t \leq b$, $v \in C$

$$
\phi(t, v)=U(t, 0)\left[x_{0}-g(v)\right]+\int_{0}^{t} U(t, s) F\left(s, \phi(s, v), \int_{0}^{s} h(s, \tau, \phi(\tau, v)) d \tau\right) d s .
$$

Define the mapping $g: X \rightarrow X$ by

$$
g(v)=\sum_{i=0}^{p} g_{i}\left(v\left(t_{i}\right)\right)
$$

Let $r>0$ and

$$
v \in C_{r}(\delta):=\left\{\phi \in C([\delta, b], X) ; \sup _{\delta \leq t \leq b}\|\phi(t, v)\| \leq r\right\}
$$

From (3.10), we have

$$
\phi(t, \tilde{v})=U(t, 0)\left[x_{0}-g(\tilde{v})\right]+\int_{0}^{t} U(t, s) F\left(s, \phi(s, \tilde{v}), \int_{0}^{s} h(s, \tau, \phi(\tau, \tilde{v}) d \tau) d s .\right.
$$


Then, for $t \in(0, b]$,

$$
\begin{aligned}
\|\phi(t, \tilde{v})\| \leq & \left\|U(t, 0)\left[x_{0}-\sum_{i=0}^{p} g_{i}\left(\tilde{v}\left(t_{i}\right)\right)\right]\right\| \\
& +\int_{0}^{t}\left\|U(t, s) F\left(s, \phi(s, \tilde{v}), \int_{0}^{s} h(s, \tau, \phi(\tau, \tilde{v})) d \tau\right)\right\| d s \\
\leq & M\left\|x_{0}-\sum_{i=0}^{p} g_{i}\left(\tilde{v}\left(t_{i}\right)\right)\right\|+M \int_{0}^{t}\left[\| F\left(s, \phi(s, \tilde{v}), \int_{0}^{s} h(s, \tau, \phi(\tau, \tilde{v})) d \tau\right)\right. \\
& -F(s, 0,0)\|+\| F(s, 0,0) \|] d s \\
\leq & M\left[\left\|x_{0}\right\|+\left\|\sum_{i=0}^{p} g_{i}\left(\tilde{v}\left(t_{i}\right)\right)\right\|\right] \\
& +M \int_{0}^{t}\left\{L\left[\|\phi(s, \tilde{v})\|+\left\|\int_{0}^{s} h(s, \tau, \phi(\tau, \tilde{v})) d \tau\right\|\right]+L_{1}\right\} d s \\
\leq & M\left[\left\|x_{0}\right\|+\sum_{i=0}^{p} \Lambda_{i}\left(\left\|\tilde{v}\left(t_{i}\right)\right\|\right)\right]+M \int_{0}^{s}\left\{L \left[\|\phi(s, \tilde{v})\|+\int_{0}^{s}[\| h(s, \tau, \phi(\tau, \tilde{v}))\right.\right. \\
& \left.-h(s, \tau, 0)\|+\| h(s, \tau, 0) \|] d \tau]+L_{1}\right\} d s \\
\leq & M\left[M_{* *}+\sum_{i=0}^{p} \Lambda_{i}(r)\right]+M L(1+N b) \int_{0}^{t} \sup _{x \in(0, b]}\|\phi(s, \tilde{v})\| d s
\end{aligned}
$$

where $M_{* *}>0$ is independent of $r$. Making use of the Gronwall's inequality, such that

$$
\sup _{t \in(0, b]}\|\phi(t, \tilde{v})\| \leq M\left[M_{* *}+\sum_{i=0}^{p} \Lambda_{i}(r)\right] e^{b M L(1+N b)} .
$$

Suppose that $\phi(t, \tilde{v}) \in C_{r}(\delta)$ is not the case for $v \in C_{r}(\delta)$. Then for some positive number $r$, there is a function $v \in C_{r}(\delta)$, but $\phi(t, \tilde{v}) \notin C_{r}(\delta)$, that is, $\sup _{t \in[\delta, b]}\|\phi(t, \tilde{v})\|>r$ for some $t \in(0, b]$. Then

$$
\begin{aligned}
1 & <\frac{1}{r} \sup _{t \in[\delta, b]}\|\phi(t, \tilde{v})\| \\
& \leq \frac{1}{r} M M_{* *} e^{b M L(1+N b)}+M e^{b M L(1+N b)} \sum_{i=0}^{p} \frac{\Lambda_{i}(r)}{r} .
\end{aligned}
$$

Using (H4)(ii) and $r$ enough large we conclude that $1<0$ which is not true. Hence there exists a positive number $r, \phi(t, \tilde{v}) \in C_{r}(\delta)$ for $v \in C_{r}(\delta)$. The proofs of the other steps are similar to those in Theorem 3.1. Therefore we omit the details. 
Remark 3.1. In Theorem 3.1, we use the condition (H3). But in Theorem 3.2, we only study a special case of the nonlocal condition namely:

$$
g(x)=\sum_{i=0}^{p} g_{i}\left(x\left(t_{i}\right)\right),
$$

where $g_{i}$ satisfies the hypothesis (H4). Then $g$ satisfies the condition (H3). In that case, we give sufficient conditions for the existence of mild solutions, which are more better than general case.

From the above proof of Theorem 3.2, we immediately obtain the following corollaries.

Corollary 3.1. Let $x_{0} \in \overline{D(A)}$. Assume that assumptions $(\mathrm{H} 1)-(\mathrm{H} 2),(\mathrm{H} 4)(\mathrm{i})$ and the following conditions are satisfied.

(H5) There exist constants $c_{i}, i=0, \ldots, p$, such that

$$
\left\|g_{i}(\phi)\right\| \leq c_{i}, \quad \phi \in C
$$

Then the nonlocal Cauchy problem (3.9) has at least one mild solution.

Corollary 3.2. Let $x_{0} \in \overline{D(A)}$. Assume that assumptions $(\mathrm{H} 1)-(\mathrm{H} 2),(\mathrm{H} 4)(\mathrm{i})$ and the following conditions are satisfied.

(H6) There exist constanrs $a_{i}$ and $b_{i}, \alpha_{i} \in[0,1), i=0, \ldots, p$, such that

$$
\left\|g_{i}(\phi)\right\| \leq a_{i}+b_{i}\|\phi\|^{\alpha_{i}}, \quad \phi \in C .
$$

Then the nonlocal Cauchy problem (3.9) has at least one mild solution.

Corollary 3.3. Let $x_{0} \in \overline{D(A)}$. Assume that assumptions $(\mathrm{H} 1)-(\mathrm{H} 2),(\mathrm{H} 4)(\mathrm{i})$ and the following conditions are satisfied.

$$
\lim _{\|\phi\| \rightarrow \infty} \sup \frac{\left\|g_{i}(\phi)\right\|}{\|\phi\|}=0, \quad i=0, \ldots, p .
$$

Then the nonlocal Cauchy problem (3.9) has at least one mild solution.

\section{Application}

To illustrate the application of the obtained results of this paper we study the following example in this section:

$$
\begin{gathered}
\frac{\partial}{\partial t} z(t, x)=a_{0}(t, x) \frac{\partial^{2}}{\partial x^{2}} z(t, x)+z(t, x)+\sin z(t, x)+\frac{1}{1+t^{2}} \int_{0}^{t} a(s) z(s, x) d s, \\
z(t, 0)=z(t, \pi)=0,
\end{gathered}
$$




$$
z(0, x)+\int_{\delta}^{1}[z(s, x)+\log (1+|z(s, x)|)] d s=z_{0}(x), \quad 0 \leq t \leq 1,0 \leq x \leq \pi,
$$

where $\delta>0, z_{0}(x) \in X=L^{2}([0, \pi])$ and $z_{0}(0)=x_{0}(\pi)=0$. Here, the functions $a_{0}(t, x)$ is continuous and is uniformly Hölder continuous in $t$.

Let $X=L^{2}([0,1])$ and the operators $A(t)$ be defined by

$$
A(t) w=a_{0}(t, x) w^{\prime \prime}
$$

with the domain $D(A)=\left\{w \in X: w, w^{\prime \prime}\right.$ are absolutely continuous, $w^{\prime \prime} \in X, w(0)=$ $w(1)=0\}$, then $A(t)$ generates an evolution system $U(t, s)$ satisfying assumptions (I) -(III) (see [12]).

We assume that the function $a(\cdot)$ is continuous on $[0,1]$, and $l=\sup _{0 \leq s \leq 1}|a(s)|<1$.

Define respectively $F:[0,1] \times X \times X \rightarrow X, h:[0,1] \times X \rightarrow X$ and $g: C([0,1], X) \rightarrow$ $\overline{D(A)}$ by

$$
\begin{gathered}
\int_{0}^{t} h(t, s, z(s))(x) d s=\frac{1}{1+t^{2}} \int_{0}^{t} a(s) z(s, x) d s \\
F\left(t, z(t), \int_{0}^{t} h(t, s, x(s)) d s\right)(x)=z(t, x)+\sin z(t, x)+\frac{1}{1+t^{2}} \int_{0}^{t} a(s) z(s, x) d s,
\end{gathered}
$$

and

$$
g(z)(x)=\int_{\delta}^{1}[z(s, x)+\log (1+|z(s, x)|)] d s, \quad z \in C([0,1], X) .
$$

Then Eq. (4.1) takes the abstract form (1.1). If we assume that $M e^{M(2+l)(1+l)}(1-\delta)<1$. From Theorem 3.1, we deduce that nonlocal Cauchy problems (4.1) has a mild solution.

\section{References}

[1] L. Byszewski, Theorems about the existence and uniquencess of a solutions of a semilinear evolution nonlocal Cauchy problem, J. Math. Anal. Appl., 162(1991), 495-505.

[2] L. Byszewski and H. Akca, Existence of solutions of a semilinear functional-differential evolution nonlocal problem, Nonlinear Anal., 34(1998), 65-72.

[3] S. K. Ntouyas and P. Ch. Tsamatos, Global existence for semilinear evolution equations with nonlocal conditions, J. Math. Anal. Appl., 210(1997), 679-687.

[4] Y. Lin and J. H. Liu, Semilinear integrodifferential equations with nonlocal Cauchy problem, Nonlinear Anal., 26(1996), 1023-1033.

[5] K. Ezzinbi and X. Fu, Existence and regularity of solutions for some neutral partial differential equations with nonlocal conditions, Nonlinear Anal., 57(2004), 1029-1041.

[6] J. Liang, J. H. Liu and T. J. Xiao, Nonlocal Cauchy problems governed by compact operator families, Nonlinear Anal. 57(2004), 183-189.

[7] S. Aizicovici and H. Lee, Nonlinear nonlocal Cauchy problems in Banach spaces, Appl. Math. Lett., 18(2005), 401-407. 
[8] S. Aizicovici and M. Mckibben, Existence results for a class of abstract nonlocal Cauchy problems, Nonlinear Anal. Ser A: Theory Methods, 39(2000), 649-668.

[9] R. Sakthivel, S. M. Anthoni and J. H. Kin, Existence and controllability result for semilinear evolution integrodifferential system, Math, Comput. Model., 41(2005), 1005-1011.

[10] W. E. Fitzgibbon, Asymptotic behavior of solutions to a class of Volterra integrodifferential equations, J. Math. Anal. Appl., 146(1990), 241-253.

[11] A. Pazy, Semigroup of Linear Operators and Applications to Partial Differential Equations, Springer-Verlag, New York, 1983.

[12] A. Freidman, Partial Differential Equations, Holt, Rinehat and Winston, New York, 1969.

Department of Mathematics, Hexi University, Zhangye, Gansu 734000, P.R.CHINA

E-mail: yanzuomao@163.com 